\title{
Synthesis of some new 4-aryloxmethylcoumarins and examination of their antibacterial and antifungal activities
}

\author{
MAHANTESHA BASANAGOUDA ${ }^{\mathrm{a}}$, MANOHAR V KULKARNI ${ }^{\mathrm{a}, *}$, DEEPAK SHARMA $^{\mathrm{b}}$, \\ VIVEK K GUPTA $^{\mathrm{b}}$, PRANESHA $^{\mathrm{c}}$, P SANDHYARANI $^{\mathrm{c}}$ and VIJAYKUMAR P RASAL ${ }^{\mathrm{c}}$ \\ ${ }^{a}$ Post-Graduate Department of Chemistry, Karnatak University, Dharwad 580003 \\ ${ }^{\mathrm{b}}$ Post-Graduate Department of Physics, University of Jammu, Jammu 180006 \\ 'Department of Pharmacology, KLE's College of Pharmacy, Belgaum 590010 \\ e-mail: manohar274@gmail.com
}

MS received 27 August 2008; revised 28 May 2009; accepted 3 June 2009

\begin{abstract}
A number of new 4-aryloxymethylcoumarins 3 and 5 have been obtained from the reaction of various 4-bromomethylcoumarins with 2-nitro- $p$-cresol 2 and 2,6-dibromo- $p$-cresol 4 . NOE studies have been carried out on two compounds to ascertain their spatial proximity. $p$-cresol ethers 7 have been subjected to bromination in chloroform and the position of bromine has been established by diffraction studies. Cleavage of the ether linkage has been observed during an attempted nitration of ethers 7 . Antimicrobial activity of all the compounds against five bacterial and five fungal species have been reported.
\end{abstract}

Keywords. 4-Bromomethylcoumarins; NOE; X-ray; electrophilic substitution; fluorescent; antimicrobial.

\section{Introduction}

Amongst all the naturally occurring oxygen heterocycles, coumarins occupy an important position in view of their wide ranging biological, ${ }^{1}$ fluorescent labelling, ${ }^{2}$ and protease inhibiting ${ }^{3}$ properties. Earlier 4-aryloxymethylcoumarins were screened as models for Claisen rearrangement. ${ }^{4}$ Importance of aryl and alkyl ether linkage in coumarins was realized with the discovery of their anti-histaminic activity. ${ }^{5}$ Antimicrobial activity, long range coupling ${ }^{6}$ and centrosymmetric nature ${ }^{7}$ of 4 -aryloxymethyl coumarins have been reported from our laboratory. Recently we have also observed that linking of biocompatible fragments like vanillin ${ }^{8}$ and paracetamol ${ }^{9}$ via an ether linkage at the allylic position in the pyran ring leads to molecular motiffs with potential antiinflammatory and dual fluorescence properties. It can be seen that 4-aryloxymethyl coumarins are associated with promising biological and structurally interesting properties.

In the present work, we have generated a number of 4-aryloxymethyl coumarins by using 2-nitro- $p$ cresol and 2,6-dibromo- $p$-cresol. NOE studies have been carried out on two compounds to ascertain

*For correspondence their spatial proximity. With a view to compare the reactivity of the phenoxy and coumarin moieties, bromination and nitration of $p$-cresol ethers 7 have been carried out. Bromination resulted in 2-bromo$p$-cresol ethers which has been proved by diffraction studies. Nitration has been found to occur in the coumarin ring with the simultaneous cleavage of the ether linkage.

\section{Experimental}

\subsection{Materials}

All the starting materials and reagents were purchased from commercial suppliers and used after further purification.

\subsection{Physical measurements}

Melting points were determined by open capillary method and are uncorrected. All the compounds were analysed satisfactorily for $\mathrm{C}, \mathrm{H}$ and $\mathrm{N}$. IR spectra $(\mathrm{KBr}$ disc) were recorded on a Nicolet-5700 FT-IR spectrophotometer. ${ }^{1} \mathrm{H}$ and ${ }^{13} \mathrm{C}$ NMR spectra were recorded on Bruker $300 \mathrm{MHz}$ spectrometer using $\mathrm{CDCl}_{3}$ as a solvent and TMS as an internal 
standard. The chemical shifts are expressed in $\delta \mathrm{ppm}$ scale down field from TMS and proton signals are indicated as $s=$ singlet, $d=$ doublet, $t=$ triplet and $m=$ multiplet. EI $70 \mathrm{EV}$ and AUTOSPEC electron Impact Mass spectrometer was used to record mass spectra. UV spectra were recorded on U-3310-UVVIS spectrophotometer and fluorescence spectra were recorded on F-7000 Fluorescence spectrometer. The NOE difference spectra were measured with an Avance Bruker $500 \mathrm{MHz}$, in $\mathrm{CDCl}_{3}$, against TMS, at $297 \mathrm{~K}$. NOE difference spectra were measured, at concentration $0.01 \mathrm{M}$, using NOE mul sequence.

\subsection{Synthesis}

2.3a Synthesis of substituted 4-bromomethylcoumarins: The required substituted 4-bromomethylcoumarins ${ }^{10} \mathbf{1}(\mathbf{a}-\mathbf{g})$ have been synthesized by the Pechmann cyclisation of various phenols with 4bromoethylacetoacetate. ${ }^{11}$

2.3b Synthesis of 2,6-dibromo-p-cresol (4) ${ }^{12}$ : The general procedure for the synthesis of coumarinyl ethers $\mathbf{3}(\mathbf{a}-\mathbf{g}), \mathbf{5}(\mathbf{a}-\mathbf{g})$ and $\mathbf{7}(\mathbf{a}-\mathbf{g})$ are as follows: A mixture of 4-methyl-2-nitro-phenol 2 (1.53 g, $10 \mathrm{mmol}$ ) and anhydrous potassium carbonate $(1.38 \mathrm{~g}, 10 \mathrm{mmol})$ was stirred for $30 \mathrm{~min}$ in dry acetone $(30 \mathrm{~mL})$. To this, 4-bromomethylcoumarin $1 \mathrm{a}$ $(2.53 \mathrm{~g}, 10 \mathrm{mmol})$ was added and the stirring was continued for $24 \mathrm{~h}$. Then, the resulting reaction mixture was poured to crushed ice. The separated solid was filtered and washed with $1: 1 \mathrm{HCl}(30 \mathrm{~mL})$ and with water. Then product 3a was recrystallised from suitable solvent.

Similar procedure was followed for the synthesis of $5(\mathbf{a}-\mathrm{g})$ and $7(\mathrm{a}-\mathrm{g})$ by the reaction of the $1(\mathbf{a}-\mathrm{g})$ with 4 and 6 respectively.

2.3c 6-Methyl-4-(4-methyl-2-nitro-phenoxymethyl)2H-chromen-2-one (3a): Blue coloured solid (acetic acid), m.p. $267^{\circ} \mathrm{C}$, yield $82 \%$; IR ( $\mathrm{KBr}, v$ in $\left.\mathrm{cm}^{-1}\right)$ 1714 (lactone $\mathrm{C}=\mathrm{O}), 1528,1345\left(\mathrm{NO}_{2}\right) ;{ }^{1} \mathrm{H}$ NMR $\left(300 \mathrm{MHz}, \mathrm{CDCl}_{3}\right): \delta 2.40\left(s, 3 \mathrm{H}, p-\mathrm{CH}_{3}\right), 2.45$ $\left(s, 3 \mathrm{H}, \mathbf{C}_{6}-\mathrm{CH}_{3}\right), 5.35\left(s, 2 \mathrm{H}, \mathbf{C}_{4}-\mathrm{CH}_{2}\right), 6.72(s, 1 \mathrm{H}$, $\left.\mathrm{C}_{3}-\mathrm{H}\right), 7.04-7.74(m, 6 \mathrm{H}, \mathrm{Ar}-\mathrm{H})$; LCMS $m / z: 326$ $[\mathrm{M}+1]$; Anal. calc. for $\mathrm{C}_{18} \mathrm{H}_{15} \mathrm{NO}_{5} ; \mathrm{C}, 66.46 ; \mathrm{H}$, 4.65 ; N, 4.31; Found: C, 66.48; H, 4.69; N, 4.36.

2.3d 7-Methyl-4-(4-methyl-2-nitro-phenoxymethyl)2H-chromen-2-one (3b): Colourless solid (acetic acid), m.p. $247^{\circ} \mathrm{C}$, yield $79 \%$; IR ( $\mathrm{KBr}, v$ in $\left.\mathrm{cm}^{-1}\right)$
1701 (lactone $\mathrm{C}=\mathrm{O}), 1532,1342\left(\mathrm{NO}_{2}\right) ;{ }^{1} \mathrm{H}$ NMR $\left(300 \mathrm{MHz}, \mathrm{CDCl}_{3}\right): \delta 2.40\left(s, 3 \mathrm{H}, p-\mathrm{CH}_{3}\right), 2.53$ $\left(s, 3 \mathrm{H}, \mathrm{C}_{6}-\mathrm{CH}_{3}\right), 5.43\left(s, 2 \mathrm{H}, \mathrm{C}_{4}-\mathrm{CH}_{2}\right), 6.91(s, 1 \mathrm{H}$, $\left.\mathrm{C}_{3}-\mathrm{H}\right), 7 \cdot 10-7 \cdot 81(\mathrm{~m}, 6 \mathrm{H}, \mathrm{Ar}-\mathrm{H})$; LCMS $m / z: 326$ $[\mathrm{M}+1]$; Anal. calc. for $\mathrm{C}_{18} \mathrm{H}_{15} \mathrm{NO}_{5} ; \mathrm{C}, 66.46 ; \mathrm{H}$, $4 \cdot 65$; N, 4.31; Found: C, 66.49; H, 4.68; N, 4.37.

\section{3e 1-(4-Methyl-2-nitro-phenoxymethyl)-benzo[f]} chromen-3-one (3c): Blue coloured solid (acetic acid), m.p. $261^{\circ} \mathrm{C}$, yield $71 \%$; IR $\left(\mathrm{KBr}, v\right.$ in $\left.\mathrm{cm}^{-1}\right)$ 1716 (lactone $\mathrm{C}=\mathrm{O}), 1531,1343\left(\mathrm{NO}_{2}\right) ;{ }^{1} \mathrm{H}$ NMR $\left(300 \mathrm{MHz}, \mathrm{CDCl}_{3}\right): \delta 2.43\left(s, 3 \mathrm{H}, p-\mathrm{CH}_{3}\right), \quad 5.88$ $\left(s, 2 \mathrm{H}, \quad \mathrm{C}_{4}-\mathrm{CH}_{2}\right), 7 \cdot 34\left(s, 1 \mathrm{H}, \quad \mathrm{C}_{3}-\mathrm{H}\right), 7 \cdot 11-8.18$ (m, 9H, Ar-H); Anal. calc. for $\mathrm{C}_{21} \mathrm{H}_{15} \mathrm{NO}_{5} ; \mathrm{C}, 69 \cdot 80$; H, 4.18; N, 3.88; Found: C, 69.83; H, 4.22; N, 3.91.

\section{$2.3 \mathrm{f}$ 4-(4-Methyl-2-nitro-phenoxymethyl)-2H-benzo}

[h]chromen-2-one (3d): Green coloured solid (acetic acid), m.p. $266^{\circ} \mathrm{C}$, yield $73 \%$; IR ( $\mathrm{KBr}, v$ in $\left.\mathrm{cm}^{-1}\right) 1705$ (lactone $\left.\mathrm{C}=\mathrm{O}\right), 1533,1345\left(\mathrm{NO}_{2}\right) ;{ }^{1} \mathrm{H}$ NMR $\left(300 \mathrm{MHz}, \mathrm{CDCl}_{3}\right): \delta 2.41\left(s, 3 \mathrm{H}, p-\mathrm{CH}_{3}\right)$, $5.53\left(s, 2 \mathrm{H}, \mathrm{C}_{4}-\mathrm{CH}_{2}\right), 7.14-8.59(m, 10 \mathrm{H}, \mathrm{Ar}-\mathrm{H})$; Anal. calc. for $\mathrm{C}_{21} \mathrm{H}_{15} \mathrm{NO}_{5} ; \mathrm{C}, 69.80 ; \mathrm{H}, 4.18 ; \mathrm{N}$, 3.88; Found: C, 69.82; H, 4.24; N, 3.90.

2.3g 6-Methoxy-4-(4-methyl-2-nitro-henoxymethyl)2H-chromen-2-one (3e): Blue coloured solid (acetic acid), m.p. $241^{\circ} \mathrm{C}$, yield $76 \%$; IR ( $\mathrm{KBr}, v$ in $\left.\mathrm{cm}^{-1}\right)$ 1701 (lactone $\mathrm{C}=\mathrm{O}), 1528,1346\left(\mathrm{NO}_{2}\right) ;{ }^{1} \mathrm{H}$ NMR $\left(300 \mathrm{MHz}, \mathrm{CDCl}_{3}\right): \delta 2.41\left(s, 3 \mathrm{H}, p-\mathrm{CH}_{3}\right), 3.95$ $\left(s, 3 \mathrm{H}, \mathrm{C}_{6}-\mathrm{OCH}_{3}\right), 5.41\left(s, 2 \mathrm{H}, \mathrm{C}_{4}-\mathrm{CH}_{2}\right), 6.97(s, 1 \mathrm{H}$, $\left.\mathrm{C}_{3}-\mathrm{H}\right), 7 \cdot 10-7.80(m, 6 \mathrm{H}, \mathrm{Ar}-\mathrm{H})$; Anal. calc. for $\mathrm{C}_{18} \mathrm{H}_{15} \mathrm{NO}_{6} ; \mathrm{C}, 63.34 ; \mathrm{H}, 4.43$; N, 4.05; Found: $\mathrm{C}$, $63 \cdot 36 ; \mathrm{H}, 4 \cdot 45 ; \mathrm{N}, 4 \cdot 09$.

2.3h 6-Chloro-4-(4-methyl-2-nitro-phenoxymethyl)$2 H$-chromen-2-one (3f): Blue coloured solid (acetic acid), m.p. $256^{\circ} \mathrm{C}$, yield $74 \%$; IR $\left(\mathrm{KBr}, v\right.$ in $\left.\mathrm{cm}^{-1}\right)$ 1715 (lactone $\mathrm{C}=\mathrm{O}), 1528,1343\left(\mathrm{NO}_{2}\right) ;{ }^{1} \mathrm{H}$ NMR $\left(300 \mathrm{MHz}, \mathrm{CDCl}_{3}\right): \delta 2.42\left(s, 3 \mathrm{H}, p-\mathrm{CH}_{3}\right), 5.40$ $\left(s, 2 \mathrm{H}, \mathrm{C}_{4}-\mathrm{CH}_{2}\right), 7.04\left(s, 1 \mathrm{H}, \mathrm{C}_{3}-\mathrm{H}\right), 7 \cdot 12-7.68$ $(m, 5 \mathrm{H}, \quad$ Ar- $\mathrm{H}), 7.84\left(s, 1 \mathrm{H}, \mathrm{C}_{5}-\mathrm{H}\right) ;{ }^{13} \mathrm{C}$ NMR $\left(\mathrm{CDCl}_{3}, 75 \mathrm{MHz}\right): 20 \cdot 4,66 \cdot 1,108 \cdot 9,112 \cdot 6,115 \cdot 7$ $116 \cdot 4,119 \cdot 5,120 \cdot 2,123 \cdot 6,127 \cdot 0,133 \cdot 5,136 \cdot 1$, $149.2, \quad 161.3,161.9,162.5$; Anal. calc. for $\mathrm{C}_{17} \mathrm{H}_{12} \mathrm{ClNO}_{5}$; C, 59.06; H, 3.50; N, 4.05; Found: C, $59 \cdot 10 ; \mathrm{H}, 3 \cdot 57 ; \mathrm{N}, 4 \cdot 07$.

$2.3 \mathrm{i}$ 6-Bromo-4-(4-methyl-2-nitro-phenoxymethyl)2H-chromen-2-one (3g): Blue coloured solid (acetic acid), m.p. $256^{\circ} \mathrm{C}$, yield $72 \%$; IR ( $\mathrm{KBr}, v$ in $\mathrm{cm}^{-1}$ ) 
1731 (lactone $\mathrm{C}=\mathrm{O}), 1533,1340\left(\mathrm{NO}_{2}\right) ;{ }^{1} \mathrm{H}$ NMR $\left(300 \mathrm{MHz}, \mathrm{CDCl}_{3}\right): \delta 2.41\left(s, 3 \mathrm{H}, p-\mathrm{CH}_{3}\right), 5.40$ $\left(s, 2 \mathrm{H}, \mathrm{C}_{4}-\mathrm{CH}_{2}\right), 7.04-7.83(m, 7 \mathrm{H}$, Ar-H); Anal. calc. for $\mathrm{C}_{17} \mathrm{H}_{12} \mathrm{BrNO}_{5} ; \mathrm{C}, 52 \cdot 33 ; \mathrm{H}, 3 \cdot 10 ; \mathrm{N}, 3 \cdot 59$; Found: C, 52.37; H, 3.14; N, 3.62.

\section{3j 6-Methyl-4-(2,6-dibromo-4-methyl-phenoxy-} methyl)-2H-chromen-2-one (5a): Colourless solid (ethylacetate), m.p. $212^{\circ} \mathrm{C}$, yield $90 \%$; IR (KBr, $v$ in $\mathrm{cm}^{-1}$ ) 1731 (lactone $\left.\mathrm{C}=\mathrm{O}\right)$; ${ }^{1} \mathrm{H}$ NMR $(300 \mathrm{MHz}$, $\left.\mathrm{CDCl}_{3}\right): \delta 2.34\left(s, 3 \mathrm{H}, p-\mathrm{CH}_{3}\right), 2.42\left(s, 3 \mathrm{H}, \mathrm{C}_{6}-\mathrm{CH}_{3}\right)$, $5 \cdot 18\left(s, 2 \mathrm{H}, \mathrm{C}_{4}-\mathrm{CH}_{2}\right), 6 \cdot 89\left(s, 1 \mathrm{H}, \mathrm{C}_{3}-\mathrm{H}\right), 7 \cdot 28-7 \cdot 39$ $(m, 5 \mathrm{H}, \mathrm{Ar}-\mathrm{H})$; LCMS $m / z: 439[\mathrm{M}+1]$; Anal. calc. for $\mathrm{C}_{18} \mathrm{H}_{14} \mathrm{Br}_{2} \mathrm{O}_{3} ; \mathrm{C}, 49 \cdot 35 ; \mathrm{H}, 3 \cdot 22$; Found: $\mathrm{C}, 49 \cdot 40$; $\mathrm{H}, 3 \cdot 30$.

\section{3k 7-Methyl-4-(2,6-dibromo-4-methyl-phenoxy-} methyl)-2H-chromen-2-one (5b): Colourless solid (ethylacetate), m.p. $185^{\circ} \mathrm{C}$, yield $92 \%$; IR (KBr, $v$ in $\mathrm{cm}^{-1}$ ) 1729 (lactone $\left.\mathrm{C}=\mathrm{O}\right)$; ${ }^{1} \mathrm{H}$ NMR $(300 \mathrm{MHz}$, $\left.\mathrm{CDCl}_{3}\right): \delta 2.33\left(s, 3 \mathrm{H}, p-\mathrm{CH}_{3}\right), 2.47\left(s, 3 \mathrm{H}, \mathrm{C}_{6}-\mathrm{CH}_{3}\right)$, $5.17\left(s, 2 \mathrm{H}, \mathrm{C}_{4}-\mathrm{CH}_{2}\right), 6.84\left(s, 1 \mathrm{H}, \mathrm{C}_{3}-\mathrm{H}\right), 7 \cdot 10$ $\left(d, 1 \mathrm{H}, J=8.0 \mathrm{~Hz}, \mathrm{C}_{6}-\mathrm{H}\right), 7.21\left(s, 1 \mathrm{H}, \mathrm{C}_{8}-\mathrm{H}\right), 7.39$ $(s, 2 \mathrm{H}, \mathrm{Ar}-\mathrm{H}) 7.47\left(d, 1 \mathrm{H}, J=8.0 \mathrm{~Hz}, \mathrm{C}_{5}-\mathrm{H}\right) ;{ }^{13} \mathrm{C}$ NMR $\left(\mathrm{CDCl}_{3}, 75 \mathrm{MHz}\right): 20 \cdot 7,22 \cdot 0,70 \cdot 0,113 \cdot 3$, $115 \cdot 3,117 \cdot 8,118 \cdot 0,123 \cdot 7,125 \cdot 8,133 \cdot 7,138 \cdot 0$, $143.4,149.9,150 \cdot 5,154 \cdot 1,161 \cdot 4$; LCMS $\mathrm{m} / z: 439$ $[\mathrm{M}+1]$; Anal. calc. for $\mathrm{C}_{18} \mathrm{H}_{14} \mathrm{Br}_{2} \mathrm{O}_{3} ; \mathrm{C}, 49.35 ; \mathrm{H}$, 3.22; Found: C, 49.42; H, 3.33.

\subsection{1-(2,6-Dibromo-4-methyl-phenoxymethyl)-}

benzo[f]chromen-3-one (5c): Colourless solid (ethylacetate), m.p. $236^{\circ} \mathrm{C}$, yield $71 \%$; IR ( $\mathrm{KBr}, v$ in $\mathrm{cm}^{-1}$ ) 1727 (lactone $\left.\mathrm{C}=\mathrm{O}\right)$; ${ }^{1} \mathrm{H}$ NMR $(300 \mathrm{MHz}$, $\left.\mathrm{CDCl}_{3}\right): \delta 2.36\left(s, 3 \mathrm{H}, p-\mathrm{CH}_{3}\right), 5.61\left(s, 2 \mathrm{H}, \mathrm{C}_{4}-\mathrm{CH}_{2}\right)$, $7.24\left(s, 1 \mathrm{H}, \mathrm{C}_{3}-\mathrm{H}\right), 7.42(s, 2 \mathrm{H}, \mathrm{Ar}-\mathrm{H}), 7.53-8.09$ ( $m, 6 \mathrm{H}$, Ar-H); Anal. calc. for $\mathrm{C}_{21} \mathrm{H}_{14} \mathrm{Br}_{2} \mathrm{O}_{3} ; \mathrm{C}$, 53.20; H, 2.98; Found: C, 53.30; H, 3.05.

$2.3 \mathrm{~m}$ 4-(2,6-Dibromo-4-methyl-phenoxymethyl)-2Hbenzo[h]chromen-2-one (5d): Colourless solid (ethylacetate), m.p. $191{ }^{\circ} \mathrm{C}$, yield $74 \%$; IR ( $\mathrm{KBr}, v$ in $\mathrm{cm}^{-1}$ ) 1718 (lactone $\left.\mathrm{C}=\mathrm{O}\right)$; ${ }^{1} \mathrm{H}$ NMR $(300 \mathrm{MHz}$, $\left.\mathrm{CDCl}_{3}\right): \delta 2 \cdot 34\left(s, 3 \mathrm{H}, p-\mathrm{CH}_{3}\right), 5 \cdot 29\left(s, 2 \mathrm{H}, \mathrm{C}_{4}-\mathrm{CH}_{2}\right)$, 7.00 (s, 1H, $\left.\mathrm{C}_{3}-\mathrm{H}\right), 7.37-7.89(\mathrm{~m}, 8 \mathrm{H}, \mathrm{Ar}-\mathrm{H})$; Anal. calc. for $\mathrm{C}_{21} \mathrm{H}_{14} \mathrm{Br}_{2} \mathrm{O}_{3} ; \mathrm{C}, 53 \cdot 20 ; \mathrm{H}, 2.98$; Found: $\mathrm{C}$, 53.32; H, 3.09.

2.3n 6-Methoxy-4-(2,6-Dibromo-4-methyl-phenoxymethyl)-2H-chromen-2-one (5e): Colourless solid (ethylacetate), m.p. $180^{\circ} \mathrm{C}$, yield $85 \%$; IR ( $\mathrm{KBr}, v$ in $\mathrm{cm}^{-1}$ ) 1710 (lactone $\left.\mathrm{C}=\mathrm{O}\right) ;{ }^{1} \mathrm{H}$ NMR $(300 \mathrm{MHz}$, $\left.\mathrm{CDCl}_{3}\right): \delta 2.34 \quad\left(s, 3 \mathrm{H}, p-\mathrm{CH}_{3}\right), 3.86 \quad(s, 3 \mathrm{H}$, $\left.\mathrm{C}_{6}-\mathrm{OCH}_{3}\right), 5 \cdot 14\left(s, 2 \mathrm{H}, \mathrm{C}_{4}-\mathrm{CH}_{2}\right), 6 \cdot 87\left(s, 1 \mathrm{H}, \mathrm{C}_{3}-\mathrm{H}\right)$, 7.15-7.39 (m, 5H, Ar-H); Anal. calc. for $\mathrm{C}_{18} \mathrm{H}_{14} \mathrm{Br}_{2} \mathrm{O}_{4} ; \mathrm{C}, 47 \cdot 61 ; \mathrm{H}, 3 \cdot 11$; Found: $\mathrm{C}, 47 \cdot 71 ; \mathrm{H}$, $3 \cdot 23$.

\subsection{6-Chloro-4-(2,6-dibromo-4-methyl-phenoxy-} methyl)-2H-chromen-2-one (5f): Colourless solid (ethylacetate), m.p. $218^{\circ} \mathrm{C}$, yield $81 \%$; IR ( $\mathrm{KBr}, v$ in $\mathrm{cm}^{-1}$ ) 1755 (lactone $\left.\mathrm{C}=\mathrm{O}\right)$; ${ }^{1} \mathrm{H}$ NMR $(300 \mathrm{MHz}$, $\left.\mathrm{CDCl}_{3}\right): \delta 2 \cdot 34\left(s, 3 \mathrm{H}, p-\mathrm{CH}_{3}\right), 5 \cdot 14\left(s, 2 \mathrm{H}, \mathrm{C}_{4}-\mathrm{CH}_{2}\right)$, $6.92\left(s, 1 \mathrm{H}, \mathrm{C}_{3}-\mathrm{H}\right), 7.33-7.65(m, 5 \mathrm{H}, \mathrm{Ar}-\mathrm{H})$; Anal. calc. for $\mathrm{C}_{17} \mathrm{H}_{11} \mathrm{Br}_{2} \mathrm{ClO}_{3} ; \mathrm{C}, 44.53 ; \mathrm{H}, 2.42$; Found: C, 44.56; H, 2.46 .

$2.3 p \quad 6-$ Bromo-4-(2,6-dibromo-4-methyl-phenoxymethyl)-2H-chromen-2-one (5g): Colourless solid (ethylacetate), m.p. $221^{\circ} \mathrm{C}$, yield $78 \%$; IR ( $\mathrm{KBr}, v$ in $\mathrm{cm}^{-1}$ ) 1755 (lactone $\left.\mathrm{C}=\mathrm{O}\right)$; ${ }^{1} \mathrm{H}$ NMR $(300 \mathrm{MHz}$, $\left.\mathrm{CDCl}_{3}\right): \delta 2 \cdot 33\left(s, 3 \mathrm{H}, p-\mathrm{CH}_{3}\right), 5 \cdot 13\left(s, 2 \mathrm{H}, \mathrm{C}_{4}-\mathrm{CH}_{2}\right)$, $6.90\left(s, 1 \mathrm{H}, \mathrm{C}_{3}-\mathrm{H}\right), 7 \cdot 27-7.80(m, 5 \mathrm{H}, \mathrm{Ar}-\mathrm{H})$; Anal. calc. for $\mathrm{C}_{17} \mathrm{H}_{11} \mathrm{Br}_{3} \mathrm{O}_{3} ; \mathrm{C}, 40.59 ; \mathrm{H}, 2 \cdot 20$; Found: $\mathrm{C}$, $40 \cdot 70 ; \mathrm{H}, 2 \cdot 28$.

$2.3 q$ 6-Methyl-4-p-tolyloxymethyl-2H-chromen-2one (7a): Colourless solid (ethanol + ethylacetate), m.p. $180^{\circ} \mathrm{C}$, yield $87 \%$; IR ( $\mathrm{KBr}, v$ in $\left.\mathrm{cm}^{-1}\right) 1718$ (lactone $\mathrm{C}=\mathrm{O}) ;{ }^{1} \mathrm{H}$ NMR $\left(300 \mathrm{MHz}, \mathrm{CDCl}_{3}\right): \delta 2.33$ $\left(s, 3 \mathrm{H}, p-\mathrm{CH}_{3}\right), 2.45\left(s, 3 \mathrm{H}, \mathrm{C}_{6}-\mathrm{CH}_{3}\right), 5.21(s, 2 \mathrm{H}$, $\left.\mathrm{C}_{4}-\mathrm{CH}_{2}\right), 6.67\left(s, 1 \mathrm{H}, \mathrm{C}_{3}-\mathrm{H}\right), 6.90(d, 2 \mathrm{H}, J=8.4 \mathrm{~Hz}$, $\operatorname{Ar}-\mathrm{H}), 7.13(d, 2 \mathrm{H}, J=8.3 \mathrm{~Hz}, \mathrm{Ar}-\mathrm{H}), 7.31-7.40(\mathrm{~m}$, $3 \mathrm{H}, \mathrm{Ar}-\mathrm{H}) ;{ }^{13} \mathrm{C} \mathrm{NMR}\left(\mathrm{CDCl}_{3}, 75 \mathrm{MHz}\right): 20 \cdot 8,21 \cdot 4$, $66 \cdot 1,113 \cdot 9,115 \cdot 1,117 \cdot 4,117 \cdot 5,123 \cdot 6,130 \cdot 5,131 \cdot 7$, $133 \cdot 3,134 \cdot 4,150 \cdot 4,152 \cdot 2,156 \cdot 0,161 \cdot 2,182 \cdot 0$; LCMS m/z: $281[\mathrm{M}+1]$; Anal. calc. for $\mathrm{C}_{18} \mathrm{H}_{16} \mathrm{O}_{3} ; \mathrm{C}$, 77.12; H, 5.75; Found: C, 77.16; H, 5.78.

2.3r 7-Methyl-4-p-tolyloxymethyl-2H-chromen-2-

one (7b): Colourless solid (ethanol + ethylacetate), m.p. $142^{\circ} \mathrm{C}$, yield $85 \%$; IR ( $\mathrm{KBr}, v$ in $\left.\mathrm{cm}^{-1}\right) 1713$ (lactone $\mathrm{C}=\mathrm{O}) ;{ }^{1} \mathrm{H}$ NMR (300 $\left.\mathrm{MHz}, \mathrm{CDCl}_{3}\right): \delta 2.32$ $\left(s, 3 \mathrm{H}, p-\mathrm{CH}_{3}\right), 2.48\left(s, 3 \mathrm{H}, \mathrm{C}_{6}-\mathrm{CH}_{3}\right), 5.20(s, 2 \mathrm{H}$, $\left.\mathrm{C}_{4}-\mathrm{CH}_{2}\right), 6.62\left(s, 1 \mathrm{H}, \mathrm{C}_{3}-\mathrm{H}\right), 6.89(d, 2 \mathrm{H}, J=8.1 \mathrm{~Hz}$, $\operatorname{Ar}-\mathrm{H}), 7.12(d, 2 \mathrm{H}, J=8.0 \mathrm{~Hz}, \operatorname{Ar}-\mathrm{H}), 7.20(s, 1 \mathrm{H}$, $\left.\mathrm{C}_{8}-\mathrm{H}\right)$, 7.28-7.49 ( $m, 2 \mathrm{H}$, Ar-H); LCMS $\mathrm{m} / z: 281$ $[\mathrm{M}+1]$; Anal. calc. for $\mathrm{C}_{18} \mathrm{H}_{16} \mathrm{O}_{3} ; \mathrm{C}, 77 \cdot 12 ; \mathrm{H}$, 5.75; Found: C, 77.18; H, $5 \cdot 77$.

\section{3s 1-(4-Tolyloxymethyl)-benzo[f]chromen-3-one}

(7c): Golden yellow Coloured solid, (ethanol + 
ethylacetate), m.p. $146^{\circ} \mathrm{C}$, yield $89 \%$; IR ( $\mathrm{KBr}, v$ in $\mathrm{cm}^{-1}$ ) 1722 (lactone $\left.\mathrm{C}=\mathrm{O}\right)$; ${ }^{1} \mathrm{H}$ NMR $(300 \mathrm{MHz}$, $\left.\mathrm{CDCl}_{3}\right): \delta 2.33\left(s, 3 \mathrm{H}, p-\mathrm{CH}_{3}\right), 5.57\left(s, 2 \mathrm{H}, \mathrm{C}_{4}-\mathrm{CH}_{2}\right)$, $6.91\left(m, 3 \mathrm{H}, \quad \mathrm{C}_{3}-\mathrm{H}\right.$ and $\left.\mathrm{Ar}-\mathrm{H}\right), 7.14(d, 2 \mathrm{H}$, $J=8.3 \mathrm{~Hz}$, Ar-H), 7.51-8.18 ( $m, 6 \mathrm{H}$, Ar-H); Anal. calc. for $\mathrm{C}_{21} \mathrm{H}_{16} \mathrm{O}_{3} ; \mathrm{C}, 79.73 ; \mathrm{H}, 5 \cdot 10$; Found: C, 79.78; H, 5.14.

$2.3 \mathrm{t}$ 4-p-Tolyloxymethyl-2H-benzo[h]chromen-2one (7d): Colourless solid (ethanol + ethylacetate), m.p. $190^{\circ} \mathrm{C}$, yield $84 \%$; IR $\left(\mathrm{KBr}, v\right.$ in $\left.\mathrm{cm}^{-1}\right) 1717$ (lactone $\mathrm{C}=\mathrm{O}) ;{ }^{1} \mathrm{H}$ NMR $\left(300 \mathrm{MHz}, \mathrm{CDCl}_{3}\right): \delta 2.33$ $\left(s, 3 \mathrm{H}, p-\mathrm{CH}_{3}\right), 5.32\left(s, 2 \mathrm{H}, \mathrm{C}_{4}-\mathrm{CH}_{2}\right), 6.78(s, 1 \mathrm{H}$, $\left.\mathrm{C}_{3}-\mathrm{H}\right), 6.93(d, 2 \mathrm{H}, J=8.3 \mathrm{~Hz}, \operatorname{Ar}-\mathrm{H}), 7.14(d, 2 \mathrm{H}$, $J=8.0 \mathrm{~Hz}, \mathrm{Ar}-\mathrm{H}), 7.58-8.62$ ( $m, 6 \mathrm{H}, \mathrm{Ar}-\mathrm{H})$; Anal. calc. for $\mathrm{C}_{21} \mathrm{H}_{16} \mathrm{O}_{3} ; \mathrm{C}, 79.73 ; \mathrm{H}, 5 \cdot 10$; Found: C, 79.76; H, 5.15.

$2.3 \mathrm{u}$ 6-Methoxy-4-p-tolyloxymethyl-2H-chromen-2one (7e): Colourless solid (ethanol + ethylacetate), m.p. $150^{\circ} \mathrm{C}$, yield $91 \%$; IR $\left(\mathrm{KBr}, v\right.$ in $\left.\mathrm{cm}^{-1}\right) 1707$ (lactone $\mathrm{C}=\mathrm{O}) ;{ }^{1} \mathrm{H}$ NMR $\left(300 \mathrm{MHz}, \mathrm{CDCl}_{3}\right): \delta 2.33$ $\left(s, 3 \mathrm{H}, p-\mathrm{CH}_{3}\right), 3.87\left(s, 3 \mathrm{H}, \mathrm{C}_{6}-\mathrm{OCH}_{3}\right), 5 \cdot 19(s, 2 \mathrm{H}$, $\left.\mathrm{C}_{4}-\mathrm{CH}_{2}\right), 6.68\left(s, 1 \mathrm{H}, \mathrm{C}_{3}-\mathrm{H}\right), 6.89-7.35(m, 7 \mathrm{H}$, Ar-H); Anal. calc. for $\mathrm{C}_{18} \mathrm{H}_{16} \mathrm{O}_{4} ; \mathrm{C}, 72.96 ; \mathrm{H}, 5.44$; Found: C, 73.02; H, 5.47.

$2.3 \mathrm{v}$ 6-Chloro-4-p-tolyloxymethyl-2H-chromen-2one (7f): Colourless solid (ethanol + ethylacetate), m.p. $180^{\circ} \mathrm{C}$, yield $87 \%$; IR $\left(\mathrm{KBr}, v\right.$ in $\left.\mathrm{cm}^{-1}\right) 1718$ (lactone $\mathrm{C}=\mathrm{O}) ;{ }^{1} \mathrm{H}$ NMR $\left(300 \mathrm{MHz}, \mathrm{CDCl}_{3}\right): \delta 2.33$ $\left(s, 3 \mathrm{H}, p-\mathrm{CH}_{3}\right), 5.17\left(s, 2 \mathrm{H}, \mathrm{C}_{4}-\mathrm{CH}_{2}\right), 6.73(s, 1 \mathrm{H}$, $\left.\mathrm{C}_{3}-\mathrm{H}\right), 6.90(d, 2 \mathrm{H}, J=8.5 \mathrm{~Hz}, \operatorname{Ar}-\mathrm{H}), 7.14(d, 2 \mathrm{H}$, $J=8.4 \mathrm{~Hz}, \mathrm{Ar}-\mathrm{H}), 7.33-7.59$ ( $m, 3 \mathrm{H}$, Ar-H); Anal. calc. for $\mathrm{C}_{17} \mathrm{H}_{13} \mathrm{ClO}_{3} ; \mathrm{C}, 67.89 ; \mathrm{H}, 4.36$; Found: C, 67.97; H, 4.40.

2.3w 6-Bromo-4-p-tolyloxymethyl-2H-chromen-2one (7g): Colourless solid (ethanol + ethylacetate), m.p. $173^{\circ} \mathrm{C}$, yield $79 \%$; IR $\left(\mathrm{KBr}, v\right.$ in $\left.\mathrm{cm}^{-1}\right) 1713$ (lactone $\mathrm{C}=\mathrm{O}) ;{ }^{1} \mathrm{H}$ NMR $\left(300 \mathrm{MHz}, \mathrm{CDCl}_{3}\right): \delta 2.33$ $\left(s, 3 \mathrm{H}, p-\mathrm{CH}_{3}\right), 5 \cdot 17\left(s, 2 \mathrm{H}, \mathrm{C}_{4}-\mathrm{CH}_{2}\right), 6 \cdot 72(s, 1 \mathrm{H}$, $\left.\mathrm{C}_{3}-\mathrm{H}\right), 6.90(d, 2 \mathrm{H}, J=6.3 \mathrm{~Hz}, \operatorname{Ar}-\mathrm{H}), 7.14(d, 2 \mathrm{H}$, $J=8.5 \mathrm{~Hz}, \mathrm{Ar}-\mathrm{H}), 7.28-7.73$ ( $m, 3 \mathrm{H}$, Ar-H); Anal. calc. for $\mathrm{C}_{17} \mathrm{H}_{13} \mathrm{BrO}_{3} ; \mathrm{C}, 59 \cdot 15 ; \mathrm{H}, 3.80$; Found: C, $59 \cdot 28 ; \mathrm{H}, 3 \cdot 84$.

\subsection{Bromination of $\mathbf{7}(\mathbf{a}-\mathbf{g})$ : General procedure}

Compound $7 \mathrm{a}(2.80 \mathrm{~g}, 10 \mathrm{mmol})$ was dissolved in $25 \mathrm{~mL}$ of chloroform or acetic acid, and to this bro- mine $(0.52 \mathrm{~mL}, 10 \mathrm{mmol})$ in $25 \mathrm{~mL}$ of chloroform or acetic acid was added with stirring, and stirring was continued for $6 \mathrm{~h}$ (reaction was monitored by TLC), the solid separated 10a was filtered, dried, and recrystallised from suitable solvent.

2.4a 6-Methyl-4-(2-bromo-4-methyl-phenoxymethyl)2H-chromen-2-one (10a): Colourless solid (ethylacetate), m.p. $228^{\circ} \mathrm{C}$, yield $80 \%$; IR ( $\mathrm{KBr}, v$ in $\mathrm{cm}^{-}$ $\left.{ }^{1}\right) 1716$ (lactone $\left.\mathrm{C}=\mathrm{O}\right) ;{ }^{1} \mathrm{H} \mathrm{NMR}\left(300 \mathrm{MHz}, \mathrm{CDCl}_{3}\right)$ : $\delta 2.31\left(s, 3 \mathrm{H}, p-\mathrm{CH}_{3}\right), 2.45\left(s, 3 \mathrm{H}, \mathrm{C}_{6}-\mathrm{CH}_{3}\right), 5.26(s$, $\left.2 \mathrm{H}, \quad \mathrm{C}_{4}-\mathrm{CH}_{2}\right), \quad 6.80 \quad\left(s, \quad 1 \mathrm{H}, \quad \mathrm{C}_{3}-\mathrm{H}\right), \quad 6.87$ $(d, 1 \mathrm{H}, J=8.2 \mathrm{~Hz}, \operatorname{Ar}-\mathrm{H}), 7.07(d, 1 \mathrm{H}, J=8.1 \mathrm{~Hz}$, Ar-H), 7.31 (s, 1H, Ar-H), 7.34-7.43 ( $m, 3 \mathrm{H}, \mathrm{Ar}-\mathrm{H})$; LCMS $m / z: 361 \quad[\mathrm{M}+2]$; Anal. calc. for $\mathrm{C}_{18} \mathrm{H}_{15} \mathrm{BrO}_{3}$; C, 60.18; H, 4.21; Found: C, 60.21; H, $4 \cdot 25$.

2.4b 7-Methyl-4-(2-bromo-4-methyl-phenoxymethyl)2H-chromen-2-one (10b): Colourless solid (ethylacetate), m.p. $196^{\circ} \mathrm{C}$, yield $73 \%$; IR ( $\mathrm{KBr}, v$ in $\left.\mathrm{cm}^{-1}\right)$ 1712 (lactone $\mathrm{C}=\mathrm{O})$ ) ${ }^{1} \mathrm{H}$ NMR $\left(300 \mathrm{MHz}, \mathrm{CDCl}_{3}\right): \delta$ $2.31\left(s, 3 \mathrm{H}, p-\mathrm{CH}_{3}\right), 2.48\left(s, 3 \mathrm{H}, \mathrm{C}_{7}-\mathrm{CH}_{3}\right), 5.25(s$, $\left.2 \mathrm{H}, \mathrm{C}_{4}-\mathrm{CH}_{2}\right), 6.75\left(s, 1 \mathrm{H}, \mathrm{C}_{3}-\mathrm{H}\right), 6.85(d, 1 \mathrm{H}$, $J=8.3 \mathrm{~Hz}, \quad$ Ar- $\mathrm{H}), \quad 7.07 \quad(d, \quad 1 \mathrm{H}, \quad J=8.2 \mathrm{~Hz}$, Ar-H), $7.13\left(d, 1 \mathrm{H}, J=8.3 \mathrm{~Hz}, \mathrm{C}_{6}-\mathrm{H}\right), 7.21(s, 1 \mathrm{H}$, Ar-H), $7.43\left(s, 1 \mathrm{H}, \mathrm{C}_{8}-\mathrm{H}\right), 7.47(d, 1 \mathrm{H}, J=8.1 \mathrm{~Hz}$, $\left.\mathrm{C}_{5}-\mathrm{H}\right) ;{ }^{13} \mathrm{C} \mathrm{NMR}\left(\mathrm{CDCl}_{3}, 75 \mathrm{MHz}\right): 20 \cdot 6,22 \cdot 0,66 \cdot 2$, $113 \cdot 1,114 \cdot 1,115 \cdot 1,117 \cdot 9,123 \cdot 4,125 \cdot 9,129 \cdot 3$, $133.5,134.6,149.8,151 \cdot 2,156 \cdot 2,160.92,181 \cdot 2$; LCMS $m / z: 361[\mathbf{M}+2] ;$ Anal. calc. for $\mathrm{C}_{18} \mathrm{H}_{15} \mathrm{BrO}_{3}$; $\mathrm{C}, 60 \cdot 18 ; \mathrm{H}, 4 \cdot 21$; Found: $\mathrm{C}, 60 \cdot 21 ; \mathrm{H}$, $4 \cdot 25$.

2.4c 1-(2-Bromo-4-methyl-phenoxymethyl)-benzo[f] chromen-3-one (10c): Colourless solid (ethylacetate), m.p. $206^{\circ} \mathrm{C}$, yield $71 \%$; IR $\left(\mathrm{KBr}, v\right.$ in $\left.\mathrm{cm}^{-1}\right)$ 1718 (lactone $\mathrm{C}=\mathrm{O})$; ${ }^{1} \mathrm{H}$ NMR (300 $\left.\mathrm{MHz}, \mathrm{CDCl}_{3}\right): \delta$ $2.33\left(s, 3 \mathrm{H}, p-\mathrm{CH}_{3}\right), 5.32\left(s, 2 \mathrm{H}, \mathrm{C}_{4}-\mathrm{CH}_{2}\right), 6.93$ $\left(m, 2 \mathrm{H}, \mathrm{C}_{3}-\mathrm{H}\right.$ and $\left.\mathrm{Ar}-\mathrm{H}\right), 7.19(d, 1 \mathrm{H}, J=7.2 \mathrm{~Hz}$, Ar-H), 7.48 ( $s, 1 \mathrm{H}, \mathrm{Ar}-\mathrm{H}), 7.77-8.68(m, 6 \mathrm{H}, \mathrm{Ar}-\mathrm{H})$; Anal. calc. for $\mathrm{C}_{21} \mathrm{H}_{15} \mathrm{BrO}_{3} ; \mathrm{C}, 63.81 ; \mathrm{H}, 3.83$; Found: C, 63.84; H, 3.85.

2.4d 4-(2-Bromo-4-methyl-phenoxymethyl)-2Hbenzo[h]chromen-2-one (10d): Colourless solid (ethylacetate), m.p. $234^{\circ} \mathrm{C}$, yield $76 \%$; IR ( $\mathrm{KBr}, v$ in $\mathrm{cm}^{-1}$ ) 1714 (lactone $\left.\mathrm{C}=\mathrm{O}\right)$; ${ }^{1} \mathrm{H}$ NMR $(300 \mathrm{MHz}$, $\left.\mathrm{CDCl}_{3}\right): \delta 2 \cdot 31\left(s, 3 \mathrm{H}, p-\mathrm{CH}_{3}\right), 5 \cdot 33\left(s, 2 \mathrm{H}, \mathrm{C}_{4}-\mathrm{CH}_{2}\right)$, $6.94\left(m, 2 \mathrm{H}, \quad \mathrm{C}_{3}-\mathrm{H}\right.$ and $\left.\mathrm{Ar}-\mathrm{H}\right), 7.11(d, 1 \mathrm{H}$, $J=7.2 \mathrm{~Hz}, \mathrm{Ar}-\mathrm{H}), 7.45(\mathrm{~s}, 1 \mathrm{H}, \mathrm{Ar}-\mathrm{H}), 7.74-8.65(\mathrm{~m}$, 
$6 \mathrm{H}, \mathrm{Ar}-\mathrm{H})$; Anal. calc. for $\mathrm{C}_{21} \mathrm{H}_{15} \mathrm{BrO}_{3} ; \mathrm{C}, 63.81 ; \mathrm{H}$, 3.83; Found: C, 63.86; H, 3.86.

\section{4e 6-Methoxy-4-(2-bromo-4-methyl-phenoxy-} methyl)-2H-chromen-2-one (10e): Colourless solid (ethylacetate), m.p. $201{ }^{\circ} \mathrm{C}$, yield $69 \%$; IR (KBr, $v$ in $\left.\mathrm{cm}^{-1}\right) 1706$ (lactone $\left.\mathrm{C}=\mathrm{O}\right)$; ${ }^{1} \mathrm{H}$ NMR $(300 \mathrm{MHz}$, $\left.\mathrm{CDCl}_{3}\right): \delta 2.31\left(s, 3 \mathrm{H}, p-\mathrm{CH}_{3}\right), 3.88\left(s, 3 \mathrm{H}, \mathrm{C}_{6}-\right.$ $\left.\mathrm{OCH}_{3}\right), 5 \cdot 24\left(s, 2 \mathrm{H}, \mathrm{C}_{4}-\mathrm{CH}_{2}\right), 6 \cdot 80\left(s, 1 \mathrm{H}, \mathrm{C}_{3}-\mathrm{H}\right)$, $6.86(d, 1 \mathrm{H}, J=8.3 \mathrm{~Hz}, \mathrm{Ar}-\mathrm{H}), 7.05-7.35(m, 4 \mathrm{H}$, $\mathrm{Ar}-\mathrm{H}), \quad 7.43 \quad\left(s, \quad 1 \mathrm{H}, \quad \mathrm{C}_{5}-\mathrm{H}\right) ; \quad$ LCMS $m / z: \quad 377$ $[\mathrm{M}+2]$; Anal. calc. for $\mathrm{C}_{18} \mathrm{H}_{15} \mathrm{BrO}_{4} ; \mathrm{C}, 57.62 ; \mathrm{H}$, 4.03; Found: C, 57.67; $\mathrm{H}, 4 \cdot 08$.

\section{4f 6-Chloro-4-(2-bromo-4-methyl-phenoxy-}

methyl)-2H-chromen-2-one (10f): Colourless solid (ethanol + ethylacetate), m.p. $202^{\circ} \mathrm{C}$, yield $76 \%$; IR $\left(\mathrm{KBr}, v\right.$ in $\mathrm{cm}^{-1}$ ) 1713 (lactone $\left.\mathrm{C}=\mathrm{O}\right) ;{ }^{1} \mathrm{H}$ NMR $\left(300 \mathrm{MHz}, \mathrm{CDCl}_{3}\right): \delta 2.31\left(s, 3 \mathrm{H}, p-\mathrm{CH}_{3}\right), 5.36$ $\left(s, 2 \mathrm{H}, \mathrm{C}_{4}-\mathrm{CH}_{2}\right), 6.81\left(s, 1 \mathrm{H}, \mathrm{C}_{3}-\mathrm{H}\right), 6.93(d, 1 \mathrm{H}$, $J=8 \cdot 1 \mathrm{~Hz}, \mathrm{Ar}-\mathrm{H}), 7 \cdot 12-7.38(m, 4 \mathrm{H}, \mathrm{Ar}-\mathrm{H}), 7.48(s$, $\left.1 \mathrm{H}, \mathrm{C}_{5}-\mathrm{H}\right)$; Anal. calc. for $\mathrm{C}_{17} \mathrm{H}_{12} \mathrm{BrClO}_{3} ; \mathrm{C}, 53.78$; H, 3.19; Found: C, 53.83; H, 3.27.

2.4g 6-Bromo-4-(2-bromo-4-methyl-phenoxymethyll)$2 \mathrm{H}$-chromen-2-one (10g): Colourless solid (ethanol + ethylacetate), m.p. $189^{\circ} \mathrm{C}$, yield $72 \%$; IR $\left(\mathrm{KBr}, v\right.$ in $\mathrm{cm}^{-1}$ ) 1709 (lactone $\mathrm{C}=\mathrm{O}$ ); ${ }^{1} \mathrm{H}$ NMR $\left(300 \mathrm{MHz}, \mathrm{CDCl}_{3}\right): \delta 2.36\left(s, 3 \mathrm{H}, p-\mathrm{CH}_{3}\right), 5 \cdot 22(s$, $\left.2 \mathrm{H}, \mathrm{C}_{4}-\mathrm{CH}_{2}\right), 6.79\left(s, 1 \mathrm{H}, \mathrm{C}_{3}-\mathrm{H}\right), 6.96(d, 1 \mathrm{H}$, $J=8.2 \mathrm{~Hz}, \mathrm{Ar}-\mathrm{H}), 7.30-7.76(\mathrm{~m}, 4 \mathrm{H}, \mathrm{Ar}-\mathrm{H}), 7.51(\mathrm{~s}$, $\left.1 \mathrm{H}, \mathrm{C}_{5}-\mathrm{H}\right)$; Anal. calc. for $\mathrm{C}_{17} \mathrm{H}_{12} \mathrm{Br}_{2} \mathrm{O}_{3} ; \mathrm{C}, 48 \cdot 15 ; \mathrm{H}$, $2 \cdot 85$; Found: C, $48 \cdot 19$; H, 2.94 .

\subsection{Nitration of $\mathbf{7}(\mathbf{a}-\mathbf{b})$ : General procedure}

Compound $7 \mathbf{a}(2.80 \mathrm{~g}, 10 \mathrm{mmol})$ was taken in $5 \mathrm{~mL}$ of concentrated sulfuric acid and cooled to $0^{\circ} \mathrm{C}$. To this, $6 \mathrm{~mL}$ of cold nitrating mixture $(2 \mathrm{~mL}$ of conc. $\mathrm{HNO}_{3}$ and $4 \mathrm{~mL}$ of conc. $\mathrm{H}_{2} \mathrm{SO}_{4}$ ) was added dropwise with stirring. Then resultant reaction mixture was poured to crushed ice and separated reddish brown colored solid 11a was filtered, washed with cold water, dried and recrystallised from ethanol.

2.5a 4-Hydroxymethyl-6-methyl-5,8-dinitro- $2 \mathrm{H}$ chromen-2-one (11a): Yellow Coloured solid (ethanol), m.p. $160^{\circ} \mathrm{C}$, yield $80 \%$; IR $(\mathrm{KBr}, v$ in $\mathrm{cm}^{-1}$ ): $3492(-\mathrm{OH}), 1738$ (lactone $\left.\mathrm{C}=\mathrm{O}\right), 1540,1345$ $\left(\mathrm{NO}_{2}\right) ;{ }^{1} \mathrm{H}$ NMR $\left(300 \mathrm{MHz}, \mathrm{DMSO}-d_{6}\right): \delta 2.49(s$, $\left.3 \mathrm{H}, \mathrm{C}_{6}-\mathrm{CH}_{3}\right), 4.80\left(s, 2 \mathrm{H}, \mathrm{C}_{4}-\mathrm{CH}_{2}\right), 5.67(s, 1 \mathrm{H}, \mathrm{OH}$,
$\mathrm{D}_{2} \mathrm{O}$ exchangeable), $6.65\left(s, 1 \mathrm{H}, \mathrm{C}_{3}-\mathrm{H}\right), 8.52(s, 1 \mathrm{H}$, $\mathrm{C}_{7}-\mathrm{H}$ ); LCMS $\mathrm{m} / \mathrm{z}$ : 279 [M-1]; Anal. calc. for $\mathrm{C}_{11} \mathrm{H}_{8} \mathrm{~N}_{2} \mathrm{O}_{7} ; \mathrm{C}, 47 \cdot 15 ; \mathrm{H}, 2 \cdot 88, \mathrm{~N}, 10 \cdot 00$; Found: C, $47 \cdot 18 ; \mathrm{H}, 2 \cdot 91, \mathrm{~N}, 10 \cdot 07$.

\section{$2.5 \mathrm{~b}$ 4-Hydroxymethyl-6-methoxy-5,8-dinitro- $2 \mathrm{H}$ -} chromen-2-one (11b): Yellow colourered solid (ethanol), m.p. $150^{\circ} \mathrm{C}$, yield $74 \%$; IR ( $\mathrm{KBr}, v$ in $\left.\mathrm{cm}^{-1}\right) 3474(-\mathrm{OH}), 1739$ (lactone $\left.\mathrm{C}=\mathrm{O}\right), 1537,1343$ $\left(\mathrm{NO}_{2}\right) ;{ }^{1} \mathrm{H}$ NMR $\left(300 \mathrm{MHz}, \mathrm{DMSO}-d_{6}\right): \delta 3.50$ $\left(s, 1 \mathrm{H},-\mathrm{OH}, \mathrm{D}_{2} \mathrm{O}\right.$ exchangeable $), 3.90 \quad(s, 3 \mathrm{H}$, $\left.\mathrm{C}_{6}-\mathrm{OCH}_{3}\right), 4.43\left(s, 2 \mathrm{H}, \mathrm{C}_{4}-\mathrm{CH}_{2}\right), 6.89\left(s, 1 \mathrm{H}, \mathrm{C}_{3}-\mathrm{H}\right)$, $8.47\left(s, 1 \mathrm{H}, \mathrm{C}_{7}-\mathrm{H}\right)$; Anal. calc. for $\mathrm{C}_{11} \mathrm{H}_{8} \mathrm{~N}_{2} \mathrm{O}_{8} ; \mathrm{C}$, 44.61; H, 2.72, N, 9.46; Found: C, 44.68; H, 2.76, $\mathrm{N}, 10 \cdot 02$.

\section{Results and discussion}

\subsection{Synthesis}

The required 4-bromomethylcoumarins 1 were prepared by the Pechmann cyclisation of substituted phenols with 4-bromoethylacetoacetate using sulphuric acid as the condensing agent. 2-nitro- $p$-cresol 2 and 2,6-dibromo- $p$-cresol 4 were used during the present work and the ethers $\mathbf{3}$ and $\mathbf{5}$ respectively were obtained under standard acetone-potassium carbonate conditions at room temperature (scheme 1). Formation of ethers was indicated by the difference in ${ }^{1} \mathrm{H}-\mathrm{NMR}$. The methylene protons in 4bromomethylcoumarins resonated at $4.6 \mathrm{ppm}$ where as the corresponding 4-aryloxymethylcoumarins exhibited this peak around $5 \cdot 2 \mathrm{ppm}$.

The IR spectrum of 6-methyl-4-(4-methyl-2-nitrophenoxymethyl)- $2 \mathrm{H}$-chromen-2-one (3a) $\left(\mathrm{R}=6-\mathrm{CH}_{3}\right)$ showed lactone carbonyl at $1714 \mathrm{~cm}^{-1}$. The ${ }^{1} \mathrm{H}-\mathrm{NMR}$ spectrum exhibited singlets at $2 \cdot 40,2.45,5.35$ and $6.72 \delta \mathrm{ppm}$ due to $p-\mathrm{CH}_{3}, \mathrm{C}_{6}-\mathrm{CH}_{3}, \mathrm{C}_{4}-\mathrm{CH}_{2}$ and $\mathrm{C}_{3}-\mathrm{H}$ respectively. The aromatic protons resonated as multiplet in the range of 7.04-7.74 $\delta \mathrm{ppm}$. This is further confirmed by its mass spectrum that shows the molecular ion peak $m / z 326(\mathbf{M}+1)$ which agrees with the molecular weight of the compound.

The IR spectrum of 6-Methyl-4-(2,6-dibromo-4methyl-phenoxymethyl)- $2 H$-chromen-2-one $\left(\mathrm{R}=6-\mathrm{CH}_{3}\right)$ exhibited lactone carbonyl at $1731 \mathrm{~cm}^{-1}$. The ${ }^{1} \mathrm{H}$-NMR spectrum showed singlets at 2.34, $2.42,5.18$ and $6.89 \delta \mathrm{ppm}$ due to $p-\mathrm{CH}_{3}, \mathrm{C}_{6}-\mathrm{CH}_{3}$, $\mathrm{C}_{4}-\mathrm{CH}_{2}$ and $\mathrm{C}_{3}-\mathrm{H}$ respectively. The remaining protons resonated as a multiplet in the range of 7.28$7.39 \delta \mathrm{ppm}$. The LCMS of the compound showed a 


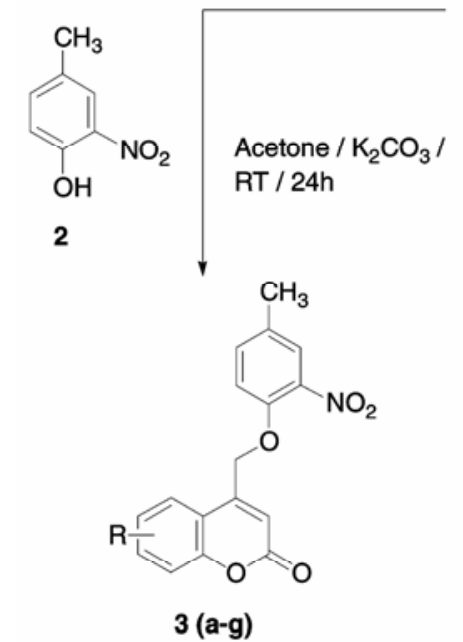

3 (a-g)<smiles>CCOC(=O)CC(=O)CBr</smiles><smiles>CCCCCCCCCOS(=O)(=O)O</smiles>

Where $1 \mathrm{a}=6-\mathrm{CH}_{3} ; 1 \mathrm{~b}=7-\mathrm{CH}_{3} ; 1 \mathrm{c}=5,6-$ Benzo; $1 \mathrm{~d}=7,8-\mathrm{Benzo} ; 1 \mathrm{e}=6-\mathrm{OCH}_{3} ; 1 \mathrm{f}=6-\mathrm{Cl} ; 1 \mathrm{~g}=6-\mathrm{Br}$

Scheme 1. Synthesis of 4-Aryloxymethylcoumarins 3 and 5.

peak at $m / z 439(\mathrm{M}+1)$ confirming its molecular weight.

The IR spectrum of 6-methyl-4-p-tolyloxymethyl$2 \mathrm{H}$-chromen-2-one $(\mathbf{7 a})^{13}\left(\mathrm{R}=6-\mathrm{CH}_{3}\right)$ exhibited lactone carbonyl at $1718 \mathrm{~cm}^{-1}$. The ${ }^{1} \mathrm{H}-\mathrm{NMR}$ spectrum showed singlets at $2.33,2.45,5.21$ and $6.67 \delta \mathrm{ppm}$ due to $p-\mathrm{CH}_{3}, \mathrm{C}_{6}-\mathrm{CH}_{3}, \mathrm{C}_{4}-\mathrm{CH}_{2}$ and $\mathrm{C}_{3}-\mathrm{H}$ respectively. The remaining aromatic protons resonated as a multiplet in the range of 6.97-7.40 $\delta \mathrm{ppm}$. The LCMS of compound showed a peak at $\mathrm{m} / \mathrm{z} 281$ $(\mathrm{M}+1)$ confirming its molecular weight.

\subsection{NOE studies}

Unambiguous assignments of methyl and aromatic protons in 4-aryloxymethylcoumarins are difficult in view of the close chemical shift values. The peri relationship between $\mathrm{H}_{5}$ and $\mathrm{CH}_{2}$ proton and the difference in multiplicity of the ortho protons related to the $\mathrm{CH}_{3}$ groups have been used to account for all the proton chemical shifts. NOE has also revealed spatial proximity between the $\mathrm{CH}_{2}$ and the ortho protons in the phenoxy moiety.

NOE studies have been carried out on two compounds $\mathbf{3 b}$ and $\mathbf{5 a}$. The $\mathrm{CH}_{2}$, two $\mathrm{CH}_{3}$ groups, aromatic protons were saturated and relative enhancements are listed in tables 1 and 2 .

In the nitro cresol ether $\mathbf{3 b}$ (figure 1), saturation of $\mathrm{C}_{4^{\prime}}-\mathrm{CH}_{3}$ at $2.37 \delta \mathrm{ppm}$ resulted strong enhancement of two signals $\mathrm{H}\left(3^{\prime}\right)(7.71, s ; 18.45 \%)$ and $\mathrm{H}$ (5') $(7.35, d, J=8.5 \mathrm{~Hz} ; 22.74 \%)$. Saturation of $\mathrm{C}_{7^{-}}$ $\mathrm{CH}_{3}$ at $2.46 \delta \mathrm{ppm}$ resulted a strong enhancement of $32.02 \%$ corresponding to two signals $\mathrm{H}(6)(7 \cdot 13, d$, $J=8.0 \mathrm{~Hz})$ and $\mathrm{H}(8)(7 \cdot 18, s)$. Saturation of $\mathrm{CH}_{2}$ at $5.31 \delta \mathrm{ppm}$ resulted significant enhancement of three signals $\mathrm{H}(3)(6.65, s ; 21.81 \%), \mathrm{H}(5)(7 \cdot 46, d$, $J=8.0 \mathrm{~Hz} ; 23.28 \%)$ and $\mathrm{H}\left(6^{\prime}\right)(7.02, d, J=8.5 \mathrm{~Hz}$; $20.48 \%$ ), and similarly saturation of aromatic protons resulted a weak enhancement of two signals, one signal of $9.84 \%$ corresponding to two methyl 
Table 1. Results of NOE difference spectra of the compound $\mathbf{3 b}$.

\begin{tabular}{|c|c|c|c|c|c|c|c|c|c|c|}
\hline${ }^{1} \mathrm{H}-\mathrm{NMR}$ & $\begin{array}{l}\mathrm{C}_{4^{\prime}}-\mathrm{CH}_{3} \\
2.37(\mathrm{~s})\end{array}$ & $\begin{array}{l}\mathrm{C}_{7^{\prime}}-\mathrm{CH}_{3} \\
2.46(\mathrm{~s})\end{array}$ & $\begin{array}{c}\mathrm{CH}_{2} \\
5 \cdot 31(\mathrm{~s})\end{array}$ & $\begin{array}{c}\mathrm{H}_{3} \\
6.65(\mathrm{~s})\end{array}$ & $\begin{array}{l}\mathrm{H}_{5} 7 \cdot 46 \\
(d, J= \\
8 \cdot 0 \mathrm{~Hz})\end{array}$ & $\begin{array}{l}\mathrm{H}_{6} 7 \cdot 13 \\
(d, J= \\
8 \cdot 0 \mathrm{~Hz})\end{array}$ & $\begin{array}{c}\mathrm{H}_{8} \\
7 \cdot 18(s)\end{array}$ & $\begin{array}{c}\mathrm{H}_{3^{\prime}} \\
7 \cdot 71(\mathrm{~s})\end{array}$ & $\begin{array}{c}\mathrm{H}_{5^{\prime}} 7 \cdot 35 \\
(d, J= \\
8 \cdot 5 \mathrm{~Hz})\end{array}$ & $\begin{array}{c}\mathrm{H}_{6}, 7 \cdot 02 \\
(d, J= \\
8 \cdot 5 \mathrm{~Hz})\end{array}$ \\
\hline $\begin{array}{l}\text { Irradiate }(2 \cdot 37) \\
\qquad \mathrm{C}_{4^{\prime}}-\mathrm{CH}_{3}\end{array}$ & $*$ & - & - & - & - & - & - & $18 \cdot 45 \%$ & $22.74 \%$ & - \\
\hline $\begin{array}{l}\text { Irradiate } \\
\qquad \mathrm{C}_{7}-\mathrm{CH}_{3}(2 \cdot 46)\end{array}$ & - & * & - & - & - & $32.02 \%$ & - & - & - & \\
\hline $\begin{array}{l}\text { Irradiate } \\
\qquad \mathrm{CH}_{2}(5 \cdot 31)\end{array}$ & - & - & $*$ & $21.81 \%$ & $23 \cdot 28 \%$ & - & - & - & - & $20.48 \%$ \\
\hline $\begin{array}{l}\text { Irradiate } \mathrm{Ar}-\mathrm{H} \\
\text { and } \mathrm{C}_{3}-\mathrm{H} \\
(6 \cdot 65-7 \cdot 71)\end{array}$ & \multicolumn{2}{|c|}{$9 \cdot 84 \%$} & $5 \cdot 62 \%$ & $*$ & $*$ & $*$ & $*$ & $*$ & $*$ & $*$ \\
\hline
\end{tabular}

*Saturated or irradiated; -, No enhancement

Table 2. Results of NOE difference spectra of the compound $\mathbf{5 a}$.

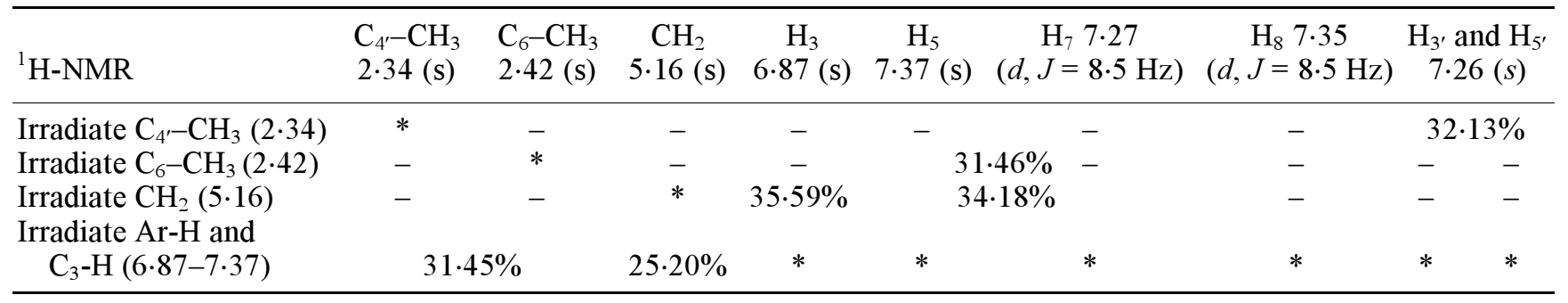

*Saturated or irradiated; -, No enhancement

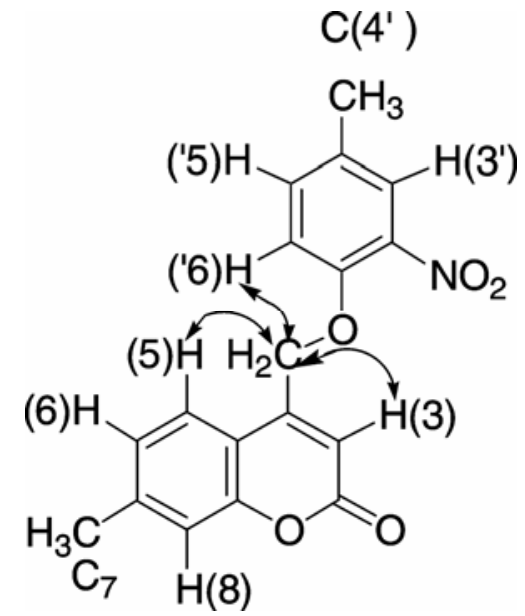

Figure 1. NOE correlation of compound $\mathbf{3 b}$.

protons $\left(\mathrm{C}_{4^{\prime}}-\mathrm{CH}_{3}, \mathrm{C}_{7}-\mathrm{CH}_{3}\right)$ and one more signal of $5.62 \%$ corresponding to $\mathrm{C}_{4}-\mathrm{CH}_{2}$ (table 1 ).

In the dibromo- $p$-cresol ether $\mathbf{5 a}$ (figure 2), saturation of $\mathrm{C}_{4^{\prime}}-\mathrm{CH}_{3}$ at $2.34 \delta \mathrm{ppm}$ resulted a strong enhancement of $32.13 \%$ corresponding to two signals $\mathrm{H}\left(3^{\prime}\right)$ and $\mathrm{H}\left(5^{\prime}\right)(7 \cdot 26, s)$. Saturation of $\mathrm{C}_{6}-\mathrm{CH}_{3}$ at $2.42 \delta \mathrm{ppm}$ resulted a strong enhancement of
$31.46 \%$ corresponding to two signals $\mathrm{H}(5)(7 \cdot 37, s)$ and $\mathrm{H}(7)(7.27, d, J=8.5 \mathrm{~Hz})$. Saturation of $\mathrm{CH}_{2}$ at $5.16 \delta \mathrm{ppm}$ resulted strong enhancement of two signals $\mathrm{H}(3)(6.87, s ; 35.59 \%)$ and $\mathrm{H}(5)(7.37, s$; $34.18 \%$ ) and similarly saturation of aromatic protons resulted in enhancement of two signals, one signal of $31.45 \%$ corresponding to two methyl protons $\left(\mathrm{C}_{4^{\prime}}-\mathrm{CH}_{3}, \mathrm{C}_{6}-\mathrm{CH}_{3}\right)$ and one more signal of $25 \cdot 20 \%$ corresponding to $\mathrm{C}_{4}-\mathrm{CH}_{2}$ (table 2).

\subsection{Electrophilic substitution}

With a view to compare the reactivity of the phenoxy moiety and the coumarin ring, bromination was attempted on ethers 7 derived from $p$-cresol and 4-bromomethylcoumarins (scheme 2). The reaction in principle can result in addition across the $\mathrm{C}_{3}-\mathrm{C}_{4}$ double bond or substitution at $\mathrm{C}-3$ position. It can also occupy at C-6 position or enter the phenoxy moiety.

The product obtained upon bromination showed the presence of $\mathrm{C}_{3}-\mathrm{H}$ in the ${ }^{1} \mathrm{H}-\mathrm{NMR}$ spectrum and no change in the IR carbonyl group of the lactone, hence addition across the $\mathrm{C}_{3}-\mathrm{C}_{4}$ double bond (8) and 


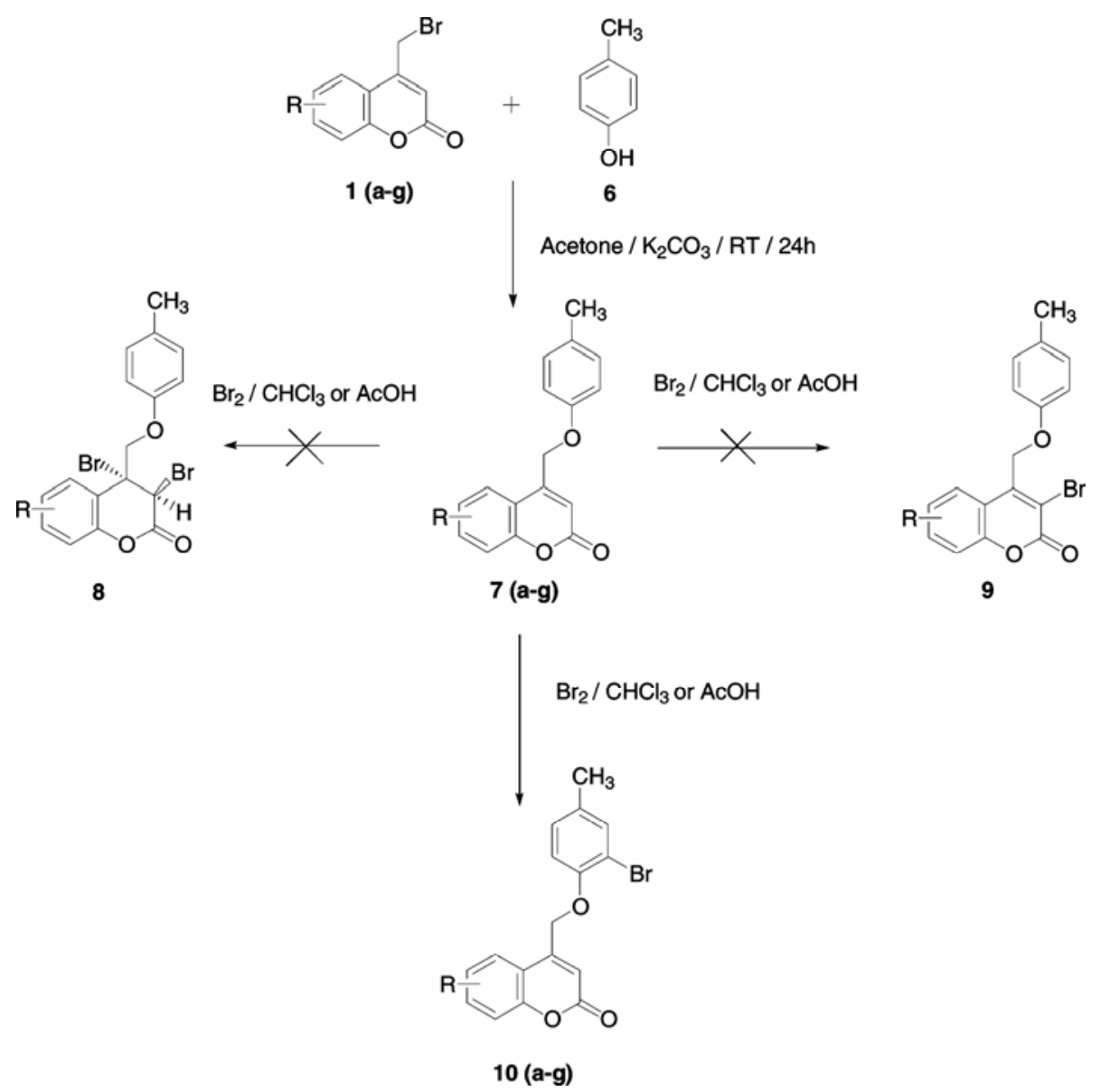

Scheme 2. Bromination of 4-Aryloxymethylcoumarins 7.

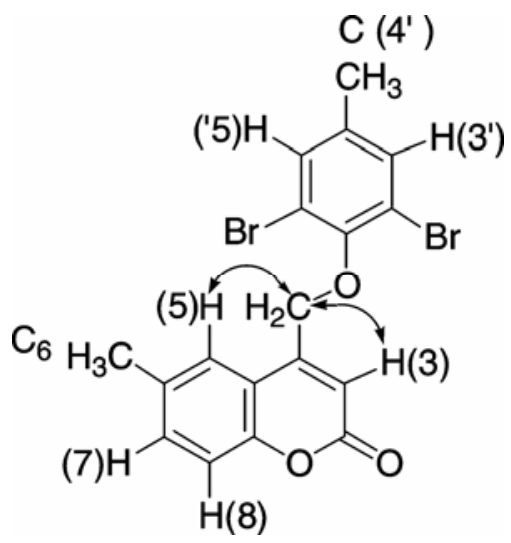

Figure 2. NOE correlation of compound $\mathbf{5 a}$.

substitution at C-3 (9) were ruled out. The mass spectrum of $10 \mathrm{~b}$ correspond to a mono brominated compound, the position of which was confirmed by diffraction studies.

Nitration of $p$-cresol ethers 7 using excess of nitrating mixture under ice-cold conditions and aqueous work-up, resulted in the formation of yellow crystalline solids. The ${ }^{1} \mathrm{H}-\mathrm{NMR}$ of these com- pounds showed the absence of one methyl group and lesser number of aromatic protons, which indicated cleavage of the ether linkage. ${ }^{14}$

The ${ }^{1} \mathrm{H}-\mathrm{NMR}$ of the ether $7 \mathbf{a}$ shows two $\mathrm{CH}_{3}$ groups at 2.33 and $2.45 \delta \mathrm{ppm}$ whereas its corresponding nitrated product shows the absence of one $\mathrm{CH}_{3}$ group. The two singlets observed at 6.65 (6.44) and $8.52(8.44)$ correspond to $\mathrm{C}_{3}-\mathrm{H}$ and $\mathrm{C}_{7}-\mathrm{H}$ respectively, which agrees with the predicted values given in parenthesis.

Nitration at C-8 is expected based on the reactivity pattern of coumarin whereas $\mathrm{C}-5$ is activated because of the electron donating groups at $\mathrm{C}-6$. The products 11a and 11b correspond to 6-substituted5,8-dinitro-4-hydroxymethyl- $2 H$-chromen-2-one (scheme 3), which is supported by the mass spectral data.

\subsection{Diffraction studies}

In order to ascertain the position of bromine formed during the bromination of ethers 7 , one of the com- 
pounds was subjected to X-ray studies. Crystals suitable for diffraction studies were grown by slow evaporation technique using ethyl acetate as the solvent. The ORTEP diagram is shown in figure 3 . The structure unambiguously shows that the bromination occurs at ortho position with respect to the ether linkage which is also expected based on the inductive effects (CCDC-695895).

\subsection{UV and fluorescence studies}

The UV and fluorescence properties of the synthesized compounds $\mathbf{3}(\mathbf{a}-\mathbf{g}), \mathbf{5}(\mathbf{a}-\mathbf{g})$ and $7(\mathbf{a}-\mathbf{g})$ were<smiles>[R]c1ccc2oc(=O)cc(COc3ccc(C)cc3)c2c1</smiles><smiles></smiles>

$11(a-b)$

$11 \mathrm{a}=\mathrm{R}=\mathrm{CH}_{3}$

$11 \mathrm{~b}=\mathrm{R}=\mathrm{OCH}_{3}$
Scheme 3. Nitration of 4-Aryloxymethylcoumarins 7 (a, e).

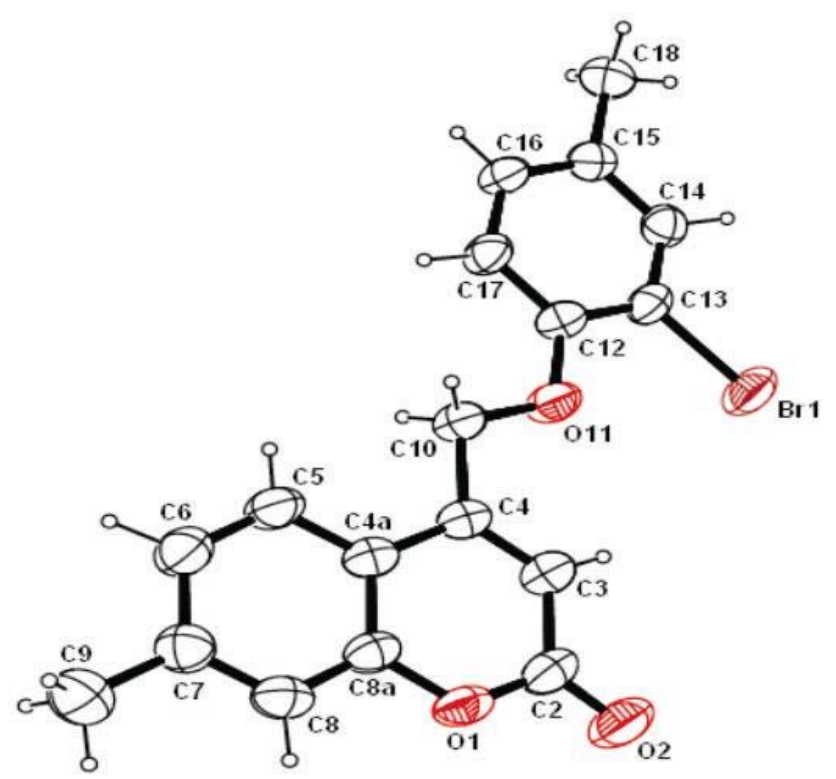

Figure 3. ORTEP diagram of compound $\mathbf{1 0 b}$. studied in chloroform. In the UV spectra, these exhibited three bands in the regions 234-246, 266-280 and $315-355 \mathrm{~nm}$, which are characteristics of 4aryloxymethyl coumarins. Introduction of bromo and nitro groups in the phenoxy moiety has not caused any significant changes in the position and intensities of the bands. In the fluorescence spectra, all the compounds showed a single band in the region of 389-443 nm, methyl compound (7b) exhibits less fluorescent with $389 \mathrm{~nm}$, whereas benzo (3d) and methoxy (3e) compounds more fluorescent with $443 \mathrm{~nm}$ each. All the compounds exhibit a Stokes shift in the range of $68-121 \mathrm{~nm}$. Among these, minimum $(68 \mathrm{~nm})$ and maximum $(121 \mathrm{~nm})$ Stoke shifts were observed for benzo (5c) and methoxy (5e) compounds respectively. In general, the maximum Stokes shift has been observed with methoxy, chloro and bromo substituents at C-6 position.

\subsection{Antimicrobial studies}

All the newly synthesized compounds $\mathbf{3}(\mathbf{a}-\mathbf{g}), \mathbf{5}(\mathbf{a}-$ g) and $7(\mathbf{a}-\mathbf{g})$ were screened for their antibacterial and antifungal activity at different concentrations of $500,250,100$ and $50 \mu \mathrm{g} / \mathrm{disc}$ by the disc diffusion method. ${ }^{15}$ The minimum inhibitory concentration (MIC) were determined by serial dilution method.

Antibacterial activity was carried out against three Gram-negative bacteria, viz. Escherichia coli, Psuedomonus aeruginosa, Klebsiella pneumoniae, and two Gram-positive bacteria, viz. Staphylococcus aureus, and Streptococcus faecalis. Ciprofloxacin was used as standard.

Antifungal activity was carried out against five fungi, viz. Aspergilus flavus, Aspergilus fumigatus, Candida albicans, Penicillium notatum and Rhizopus. Fluconazole was used as standard.

The investigation of antibacterial screening data (table 3) revealed that all the tested compounds showed moderate to good bacterial inhibition. Compounds $7 \mathrm{f}$ and $7 \mathrm{~g}$ were found to be very active against $E$. coli. The benzo compound (7d) is highly active against $P$. aeruginosa, whereas the compounds $3 \mathbf{e}, 3 \mathbf{3}, \mathbf{7 b}$ and $7 \mathbf{g}$ are more active against $K$. pneumoniae. Compounds with methyl groups (5b) and (7a) exhibited high activity against $S$. aureus. For $S$. faecalis, $7 \mathbf{f}$ was equipotent compared to standard drug Ciprofloxacin. In general, the compounds possessing methoxy (e), chloro (f), bromo (g) substituents at C6-position of coumarin showed higher activity compared to the remaining against both Gram-positive and Gram-negative bacteria. 
Table 3. Results of antibacterial activity of the compounds $3(\mathbf{a}-\mathbf{g}), \mathbf{5}(\mathbf{a}-\mathbf{g})$ and 7 (a-g) at concentration of $50 \mu \mathrm{g} / \mathrm{disc}$.

\begin{tabular}{|c|c|c|c|c|c|}
\hline \multirow[b]{2}{*}{ Compound } & \multicolumn{5}{|c|}{ Diameter of the zone of inhibition in $\mathrm{mm}$ (relative inhibition \%) } \\
\hline & E. coli & P. aeruginosa & K. pneumoniae & S. aureus & S. faecalis \\
\hline $3 \mathbf{a}$ & $8(44 \cdot 4)$ & $12(48)$ & $11(55)$ & $9(47 \cdot 3)$ & $15(75)$ \\
\hline 3b & $10(55 \cdot 6)$ & $16(64)$ & $12(60)$ & $12(63 \cdot 1)$ & $12(60)$ \\
\hline $3 c$ & $9(50)$ & $12(48)$ & $12(60)$ & $9(47 \cdot 3)$ & $11(55)$ \\
\hline 3d & $7(38 \cdot 9)$ & $12(48)$ & $12(60)$ & $8(42 \cdot 1)$ & $12(60)$ \\
\hline $3 e$ & $10(55 \cdot 6)$ & $16(64)$ & $18(90)$ & $11(57.9)$ & $12(60)$ \\
\hline $3 f$ & $9(50)$ & $12(48)$ & $17(85)$ & $10(52 \cdot 6)$ & $10(50)$ \\
\hline $3 g$ & $12(66 \cdot 7)$ & $18(72)$ & $14(70)$ & $14(73.7)$ & $12(60)$ \\
\hline $5 a$ & $9(50)$ & $12(48)$ & $11(55)$ & $10(52 \cdot 6)$ & $10(50)$ \\
\hline $5 b$ & $11(61 \cdot 1)$ & $12(48)$ & $13(65)$ & $17(89 \cdot 5)$ & $13(65)$ \\
\hline $5 c$ & $8(44 \cdot 4)$ & $9(36)$ & $10(50)$ & $10(52 \cdot 6)$ & $12(60)$ \\
\hline $5 d$ & $11(61 \cdot 1)$ & $10(40)$ & $14(70)$ & $15(78.9)$ & $16(80)$ \\
\hline $5 e$ & $9(50)$ & $10(40)$ & $9(45)$ & $15(78.9)$ & $13(65)$ \\
\hline $5 f$ & $12(66 \cdot 7)$ & $16(64)$ & $16(80)$ & $11(57.9)$ & $14(70)$ \\
\hline $5 g$ & $10(55 \cdot 6)$ & $16(64)$ & $14(70)$ & $9(47 \cdot 3)$ & $15(75)$ \\
\hline $7 \mathbf{a}$ & $9(50)$ & $18(72)$ & $16(80)$ & $16(84 \cdot 2)$ & $13(65)$ \\
\hline $7 b$ & $13(72 \cdot 2)$ & $14(56)$ & $18(90)$ & $12(63 \cdot 1)$ & $12(60)$ \\
\hline $7 c$ & $11(61 \cdot 1)$ & $16(64)$ & $12(60)$ & $9(47 \cdot 3)$ & $13(65)$ \\
\hline $7 d$ & $10(55 \cdot 6)$ & $20(80)$ & $14(70)$ & $9(47 \cdot 3)$ & $13(65)$ \\
\hline $7 e$ & $13(72 \cdot 2)$ & $12(48)$ & $14(70)$ & $10(52 \cdot 6)$ & $15(75)$ \\
\hline $7 f$ & $14(77 \cdot 8)$ & $15(60)$ & $16(80)$ & $12(63 \cdot 1)$ & $20(100)$ \\
\hline $7 g$ & $14(83 \cdot 3)$ & $15(60)$ & $18(90)$ & $13(68.4)$ & $12(60)$ \\
\hline Ciprofloxacin $10 \mu \mathrm{g} / \mathrm{disc}$ & $18(100)$ & $25(100)$ & $20(100)$ & $19(100)$ & $20(100)$ \\
\hline
\end{tabular}

Table 4. Results of antifungal activity of the compounds $3(\mathbf{a}-\mathbf{g}), \mathbf{5}(\mathbf{a}-\mathbf{g})$ and $7(\mathbf{a}-\mathbf{g})$ at concentration of $50 \mu \mathrm{g} / \mathrm{disc}$.

\begin{tabular}{|c|c|c|c|c|c|}
\hline \multirow[b]{2}{*}{ Compound } & \multicolumn{5}{|c|}{ Diameter of the zone of inhibition in $\mathrm{mm}$ at concentration of $50 \mu \mathrm{g} /$ disc (relative inhibition $\%$ ) } \\
\hline & A. flavus & A. fumigatus & C. albicans & P. notatum & Rhizopus \\
\hline $3 \mathbf{a}$ & $12(40)$ & $16(66 \cdot 7)$ & $13(54 \cdot 1)$ & $14(53 \cdot 8)$ & $18(69 \cdot 2)$ \\
\hline $3 \mathbf{b}$ & $30(100)$ & $24(100)$ & $12(50)$ & $12(46 \cdot 1)$ & $14(53 \cdot 8)$ \\
\hline $3 \mathbf{c}$ & $16(53 \cdot 3)$ & $16(66 \cdot 7)$ & $13(54 \cdot 1)$ & $20(76 \cdot 9)$ & $26(100)$ \\
\hline 3d & $20(66 \cdot 7)$ & $24(100)$ & $13(54 \cdot 1)$ & $12(46 \cdot 1)$ & $14(53 \cdot 8)$ \\
\hline $3 e$ & $28(93 \cdot 3)$ & $22(91 \cdot 7)$ & $11(45 \cdot 8)$ & $12(46 \cdot 1)$ & $13(50)$ \\
\hline $3 f$ & $20(66 \cdot 7)$ & $24(100)$ & $11(45 \cdot 8)$ & $12(46 \cdot 1)$ & $14(53 \cdot 8)$ \\
\hline $3 g$ & $14(46 \cdot 7)$ & $20(83 \cdot 3)$ & $12(50)$ & $14(53 \cdot 8)$ & $18(69 \cdot 2)$ \\
\hline $5 a$ & $11(36 \cdot 7)$ & $12(50)$ & $10(41 \cdot 7)$ & $9(34 \cdot 6)$ & $22(84 \cdot 6)$ \\
\hline $5 b$ & $10(33 \cdot 3)$ & $18(75)$ & $8(33 \cdot 3)$ & $24(92 \cdot 3)$ & $14(53 \cdot 8)$ \\
\hline $5 c$ & $20(66 \cdot 7)$ & $20(83 \cdot 3)$ & $14(58 \cdot 3)$ & $26(100)$ & $16(61 \cdot 5)$ \\
\hline 5d & $17(56 \cdot 7)$ & $24(100)$ & $18(75)$ & $23(88 \cdot 5)$ & $22(84 \cdot 6)$ \\
\hline $5 e$ & $11(36 \cdot 7)$ & $12(50)$ & $16(66 \cdot 7)$ & $22(84 \cdot 6)$ & $24(92 \cdot 3)$ \\
\hline $5 f$ & $13(43 \cdot 3)$ & $18(75)$ & $14(58 \cdot 3)$ & $16(61 \cdot 5)$ & $22(84 \cdot 6)$ \\
\hline $5 g$ & $12(40)$ & $24(100)$ & $14(58 \cdot 3)$ & $16(61 \cdot 5)$ & $20(76 \cdot 9)$ \\
\hline $7 \mathbf{a}$ & $9(30)$ & $18(75)$ & $8(33 \cdot 3)$ & $14(53 \cdot 8)$ & $14(53 \cdot 8)$ \\
\hline $7 b$ & $26(86 \cdot 7)$ & $22(91 \cdot 7)$ & $16(66 \cdot 7)$ & $14(53 \cdot 8)$ & $18(69 \cdot 2)$ \\
\hline $7 c$ & $12(40)$ & $24(100)$ & $11(45 \cdot 8)$ & $10(38 \cdot 5)$ & $12(46 \cdot 1)$ \\
\hline $7 d$ & $9(30)$ & $20(83 \cdot 3)$ & $24(100)$ & $18(69 \cdot 2)$ & $12(46 \cdot 1)$ \\
\hline $7 e$ & $10(33 \cdot 3)$ & $8(33 \cdot 3)$ & $10(41 \cdot 7)$ & $12(46 \cdot 1)$ & $20(76 \cdot 9)$ \\
\hline $7 f$ & $12(10)$ & $16(66 \cdot 7)$ & $12(50)$ & $9(34 \cdot 6)$ & $24(92 \cdot 3)$ \\
\hline $7 \mathrm{~g}$ & $11(36 \cdot 7)$ & $14(58 \cdot 3)$ & $12(50)$ & $23(88 \cdot 5)$ & $20(76 \cdot 9)$ \\
\hline Fluconazole $30 \mu \mathrm{g} / \mathrm{disc}$ & $30(100)$ & $24(100)$ & $24(100)$ & $26(100)$ & $26(100)$ \\
\hline
\end{tabular}


Similarly, the screening data of antifungal activity revealed that all the tested compounds showed moderate to good fungicidal activity. Compounds $\mathbf{3 b}, \mathbf{3 e}$ and $7 \mathbf{b}$ were more active against $A$. flavus, among these $\mathbf{3 b}$ was equipotent compared to standard drug. Compounds 3b, 3d, 3f, 5d, 5g and 7c were equipotent to standard drug against $A$. fumigatus. The benzo compound (7d) was equipotent compared to standard drug against $C$. albicans whereas for $P$. notatum, $\mathbf{5 b}$ and $\mathbf{5 c}$ were more active, among these $\mathbf{5 c}$ was equipotent compared to standard drug. Compounds 3c, 5e and 7f were more active against Rhizopus. In general, the compounds with 5,6-benzo (c) and 7,8-benzocoumarins (d) have shown equipotent activities compared to standard drug Fluconazole. The results of investigated compounds are summarized in table 4 .

\section{Conclusion}

In conclusion, we have generated a number of nitro and dibromo-4-aryloxymethylcoumarins, the former possessing promising antifungal activity. We have also studied the reactivity of phenoxy and coumarin moieties towards hard and soft electrophiles. Bromination resulted in substitution in the phenoxy moiety whereas nitration occurred in the coumarin ring with simultaneous cleavage of the ether linkage.

\section{Acknowledgements}

Authors thank the University Sophisticated Instrumentation Centre (USIC), Karnatak University, Dharwad for the spectral data. Thanks are also due to Professor CM Sun, Department of Applied Chemistry, National Chiao-Tung University, Hsinchu, Taiwan for providing difference NOE spectral data. One of the authors, Mr Mahantesha Basanagouda is grateful to Karnatak University, Dharwad for a University Research Studentship.

\section{References}

1. (a) For review on coumarins, see: Kulkarni M V, Kulkarni G M, Lin C H and Sun C M 2006 Curr. Med. Chem. 13 2795; (b) Fylaktakidou K C, Hadjpavlou D, Litinas K E and Nicolaides D N 2004 Curr. Pharm. Design 103813

2. (a) Wang W and Li H 2004 Tetrahedron Lett. 45 8479; (b) Fernandes M J G, Goncalves M S T and Costa S P G 2008 Tetrahedron 643032

3. (a) Frederick R, Charlier C, Robert S, Wouters J, Masereel B and Pochet L 2006; Bioorg. Med. Chem. Lett. 16 2017; (b) Garino C, Pietrancosta N, Laras Y, Moret V, Rolland A, Quelever G and Kraus J L 2006 Bioorg. Med. Chem. Lett. 161995

4. Thyagarajan B S, Balasubramanian K K and Bhima Rao R 1967 Tetrahedron 231893

5. Buckle D R, Outred D J, Ross J W, Smith H, Smith R J, Spicer B A and Gasson B C 1979 J. Med. Chem. 22158

6. Kulkarni M V, Pujar B J and Patil V D 1983 Arch. Pharm. 31615

7. Vasudevan K T, Kulkarni M V and Pauutaraja 1994 Acta Cryst. C50 1286

8. Ghate M D, Kulkarni M V, Shobha R and Kattimani S Y 2003 Eur. J. Med. Chem. 38297

9. Shastri L A, Ghate M D and Kulkarni M V 2004 Indian J. Chem. B43 2416

10. Kulkarni M V and Patil V D 1981 Arch. Pharm. 314 708

11. Burger A and Ullyot G E 1947 J. Org. Chem. 12346

12. Ananthakrishnan S and Chandalia S B 2006 Org. Process. Res. Dev. 10487

13. Kulkarni M V, Pujar B G and Patil V D 1981 Arch. Pharm. 31615

14. Jaques D and Leisten J A 1961 J. Chem. Soc. 4963

15. Koneman E W, Allen S D, Janda W M, Schreckenberger P C and Washington Jr C W 1997 Colour atlas and textbook of diagnostic microbiology (Philadelphia: Lippincott-Raven Publishers) Chapter 15, Antibacterial susceptibility testing, 5 th edn, pp. 785-844 